\author{
Franco Mongini \\ Giancarlo Castellano \\ Andrea Deregibus \\ Eugenia Rota \\ Annalisa Bosco
}

\section{Cortical involvement during the description of head pain}

Received: 1 June 2006

Accepted in revised form: 17 August 2006

Published online: 25 October 2006

F. Mongini (区) • A. Deregibus • E. Rota

A. Bosco

Headache and Facial Pain Unit

Department of Clinical Pathophysiology

University of Turin

Corso Dogliotti 14, I-10126 Torino, Italy

e-mail: franco.mongini@unito.it

G. Castellano

Nuclear Medicine Unit

University of Turin

Corso Dogliotti 14, I-10126 Torino, Italy

\begin{abstract}
Pain perception involves several cortical areas. Our purpose was to examine cortical activity in patients describing their cephalic pain with the MacGill Pain Questionnaire (MPQ). Two SPECT analyses were performed in pain-free periods in 10 patients with migraine $(n=8)$ or myogenous facial pain $(n=2)$. The MPQ was administered in the first session, while no task was to be performed during the second session. Differences were calculated using statistical parametric mapping (SPM99), also taking the MPQ pain rating index (PRI) as covariance.
\end{abstract}

During the MPQ session, clusters of activation were observed in the orbitofrontal cortex, the insula and the anterior cingulate cortex (ACC) of the left brain, but not significantly so. Using the MPQ PRI as covariate, significant areas of activation were found in the left frontal lobe, the Brodmann area 32 and in the ACC. The description of pain seems to activate cortical areas similar to those involved in actual pain perception.

Keywords Head pain • Pain description - MPQ - Cortical activation - SPECT

\section{Introduction}

Several imaging studies have shown that, besides the somatosensory cortex, brain processing of pain involves different cortical areas and, in particular, the anterior cingulate cortex (ACC), the orbitofrontal cortex (OFC) and the prefrontal cortex [1, 2]. In two recent studies [3, 4] aimed at investigating the subjective reality of pain, functional magnetic resonance imaging revealed significant changes during a hypnotically induced pain experience - in the absence of any noxious stimulus - in the ACC, thalamus, insula, prefrontal and parietal cortices: this activation pattern was similar to that observed during pain from nociceptive sources.

Less attention has been given so far to the brain activity consequent to pain description and recall.

By far the best known and most widely employed verbal methodology to recall and describe pain is the MacGill Pain Questionnaire (MPQ) [5]. It consists of 20 sets of qualitative and quantitative verbal descriptors, conceived to measure the sensory, affective and evaluative dimensions of pain. Descriptors can be examined individually and a pain rating index (PRI) can be calculated.

The purpose of this preliminary study was to examine the cortical activity of patients affected by recurrent head pain when asked to describe their pain sensations by means of the MPQ.

\section{Methods}

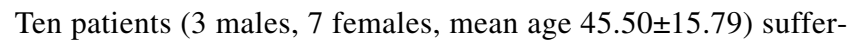
ing from episodic migraine $(n=8)$ or acute myogenous facial pain $(n=2)$ referred to the Headache and Facial Pain Unit, the Department of Clinical Pathophysiology, University of Turin were enrolled. The diagnoses were made according to the IHS criteria [6]. All subjects were right-handed, as assessed by the Edinburgh Handedness Inventory [7]. The mean total PRI were not statistically different between subjects affected by myoge- 
nous facial pain and by migraine; the pain frequency was similar (2-5 per month) for both types of headache: therefore, the two diagnostic groups were merged and examined together. The study protocol was approved by the Ethics Committee of our hospital and informed consent was obtained from all patients.

Two SPECT analyses were performed in pain-free periods. During both sessions the patients were sitting in a quiet room with the eyes covered. Eight hundred MBq of 99mTc-ECD (Neurolite, Dupont Radiopharmaceuticals, Brussels, Belgium) was injected through the venous access. About 45 minutes after radiopharmaceuticals injection, SPECT images were obtained with a two-head gamma-camera (Neurolite, Dupont Radiopharmaceuticals) fitted with fanbeam collimators. We obtained 120 frames of 1282 pixels over a $360^{\circ}$ orbit with a total acquisition time of $20 \mathrm{~min}$. In the first session patients were instructed to choose between the descriptors of the MPQ, read aloud by the clinician. The task was completed within one minute after the injection of $99 \mathrm{mTc}-\mathrm{ECD}$ had started. The second session was performed within $48 / 72 \mathrm{~h}$ of the first. During this session, the patients were simply resting and had no task to perform; neither instructions, nor verbal stimuli were given.

SPECT reconstruction was performed with the General Electric Xpert V5.5 computer, using a Metz filter (cut-off 0.43 cycle/cm and power 30); attenuation correction was performed with Chang's uniform attenuation method and attenuation coefficient was set to 0.11 $\mathrm{cm}^{-1}$. Tomographic resolution of the system was about $10 \mathrm{~mm}$. After reconstruction, 15 transaxial slices, voxel size $1.72 \times 1.72 \times 8.6 \mathrm{~mm}^{3}$, were converted from DICOM to Interfile format. Images were transferred to a Windows XP Professional computer and converted to analyse format with MRIcro application. Images were then analysed by SPM99 (Wellcome Department of Cognitive Neurology, London, UK). SPM analysis was performed with Matlab 6.1 (MathWorks Inc., Natick, MA, USA). Images were pre-processed applying the coregister and reslice option and resampled using a sinc interpolation method. The obtained images were then normalised to the SPM99 SPECT template with a resulting voxel size of $2 \times 2 \times 2$

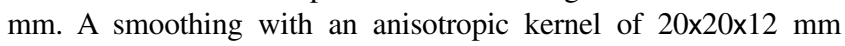
according to our FWHM resolution was done for all the images.

Scan differences in global activity were adjusted for by proportional or by grand mean scaling according to the ANOVA or ANCOVA adopted studies, respectively. A grey matter threshold of 0.8 was then used. A statistical parametric mapping (SPM99) [8] was applied to determine regions of increased activity when the MPQ was administered. Statistical comparisons between conditions were performed using $t$-statistics. The data from the MPQ obtained in the first session were assessed. The total PRI of the MPQ was calculated for each patient and statistical comparisons between the two SPECT analyses were repeated using the total PRI obtained from the MPQ as covariate.

Table 1 Volume summary ( $p$-value corrected for entire volume) of MPQ session versus rest, taking the total PRI obtained from the MPQ as covariate

\begin{tabular}{|c|c|c|c|c|}
\hline \multicolumn{2}{|c|}{ Cluster-level } & \multicolumn{2}{|c|}{ Voxel-level } & \multirow[t]{2}{*}{$x, y, z(\mathrm{~mm})$} \\
\hline$p$ corrected & $p$ uncorrected & $p$ corrected & $p$ uncorrected & \\
\hline \multirow[t]{3}{*}{0.044} & 0.002 & 0.992 & 0.001 & $-14,38,6^{*}$ \\
\hline & & 0.999 & 0.002 & $-16,32,16^{*}$ \\
\hline & & 1.000 & 0.003 & $-20,24,10^{*}$ \\
\hline \multirow[t]{3}{*}{1.000} & 0.319 & 1.000 & 0.006 & $-26,-44,16$ \\
\hline & & 1.000 & 0.009 & $-22,-48,10$ \\
\hline & & 1.000 & 0.024 & $-32,-46,30$ \\
\hline \multirow[t]{3}{*}{1.000} & 0.488 & 1.000 & 0.015 & $24,8,24$ \\
\hline & & 1.000 & 0.018 & $30,24,8$ \\
\hline & & 1.000 & 0.022 & $26,12,16$ \\
\hline \multirow[t]{2}{*}{1.000} & 0.503 & 1.000 & 0.022 & $-8,-32,8$ \\
\hline & & 1.000 & 0.041 & $-12,-22,8$ \\
\hline 1.000 & 0.976 & 1.000 & 0.035 & $18,4,18$ \\
\hline 1.000 & 0.976 & 1.000 & 0.037 & $-34,20,14$ \\
\hline 1.000 & 0.950 & 1.000 & 0.040 & $24,14,42$ \\
\hline 1.000 & 0.962 & 1.000 & 0.043 & $10,56,36$ \\
\hline 1.000 & 0.962 & 1.000 & 0.044 & $20,4,14$ \\
\hline 1.000 & 0.976 & 1.000 & 0.044 & $-44,20,12$ \\
\hline 1.000 & 0.976 & 1.000 & 0.044 & $10,50,38$ \\
\hline 1.000 & 0.976 & 1.000 & 0.044 & $6,42,40$ \\
\hline 1.000 & 0.976 & 1.000 & 0.044 & $24,4,16$ \\
\hline 1.000 & 0.962 & 1.000 & 0.045 & $-36,52,24$ \\
\hline 1.000 & 0.976 & 1.000 & 0.045 & $8,48,42$ \\
\hline 1.000 & 0.976 & 1.000 & 0.046 & $-32,48,20$ \\
\hline
\end{tabular}

* -14, 38, 6: left celebrum, anterior cingulate, grey matter, Broadmann area 32; -16, 32, 16: left celebrum, limbic lobe, anterior cingulate, grey matter, Broadmann area 32; -20, 24, 10: left celebrum, sub-lober, claustrum, grey matter, according to Tailarach lobels 


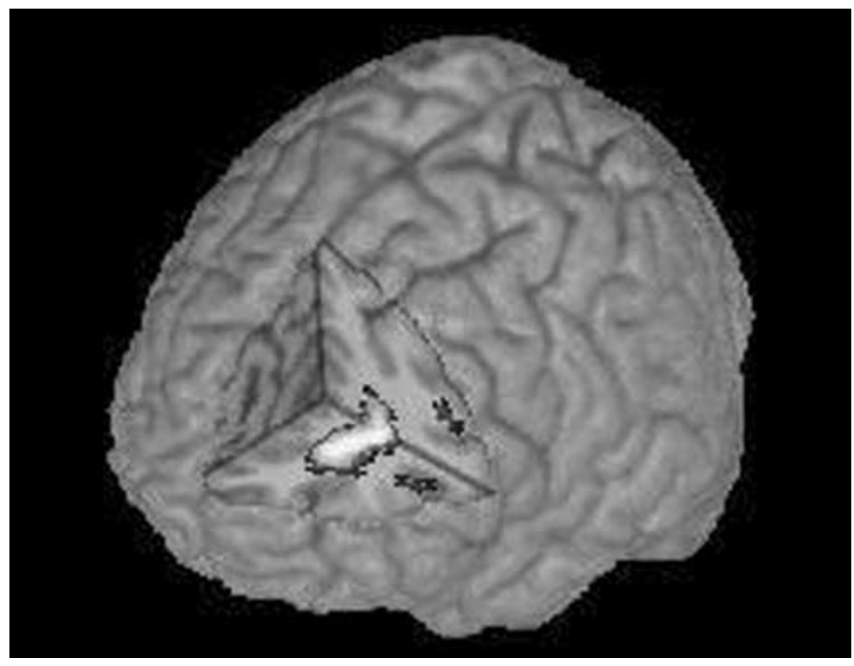

Fig. 1 With the MPQ total index as covariance, significant areas of activation are found in the anterior cingulate, the left limbic lobe and the Brodmann area 32 (see Table 1)

\section{Results}

Comparison of SPECT data in the two conditions showed several clusters of activation during the administration of the MPQ with respect to the condition of rest. They included Broadman areas 45, 47 (OFC), the insula and the ACC of the left brain. However, in none of these areas was a statistically significant level reached. While using the total PRI as covariate, significant $(p=0.044)$ areas of activation were found in the ACC, the left limbic lobe and the Brodmann area 32 (Table 1 and Figure 1).

\section{Discussion}

To the best of our knowledge, this is the first study to investigate brain activity during a task involving description of pain by patients during a pain-free period. Clusters of activation in the ACC and the OFC were observed as a consequence of the administration of the MPQ. The objection may be raised that the same cortical areas could be activated if the subjects were asked to recall and describe events different from a pain experience. However, these areas are most consistently involved in pain functional neuroimaging studies and are part of the medial pain system concerned with the emotional and motivational processing of pain. In particular, pain unpleasantness is main- ly encoded in the ACC [9]. Then, anticipation of pain may, itself, induce changes in brain nociceptive networks [10]. Moreover, recent experimental evidence has been provided, by means of functional magnetic resonance imaging, of a strict similarity in the processing of both hypnotically and physically induced experience of pain; the subjective dimension of pain (in the absence of any noxious stimulus) seems to involve brain regions (ACC, thalamus, insula, cerebellum, prefrontal and parietal cortices) variously described as belonging to the pain network [3, 4]. Higher levels of activation were found with physically induced pain with respect to hypnotically induced pain, maybe owing to the more intense pain experience in the former condition [3, 4]. In our research, the clusters of activation during description of head pain agree very closely with the results of the aforementioned studies [3, 4], providing further evidence that brain regional activation within the pain circuitry is specifically involved in the subjective reality of pain.

While mere imagining pain (the presence of a noxious heat stimulus) yielded only a minimal activation of the pain network in the study of Derbyshire et al. [3], our preliminary findings (which need to be confirmed) suggest that remembering and describing the subjective experience of a recurrent pain may enhance cortical involvement, maybe through stronger emotional mechanisms.

In a neuropsychological study aimed at assessing the function of the frontal lobe in female patients with chronic migraine, Mongini et al. [11] found a change in the functioning of the prefrontal and orbitofrontal cortices, similar to those observed in psychiatric conditions (in particular, in obsessive-compulsive disorders), which seems to confirm, also from this perspective, the role of prefrontal and orbitofrontal cortices in head pain.

Obviously, some shortcomings affect the present, preliminary study. First, the sample size is small: this may appear even more evident if the clinical heterogeneity of migraine is considered. Also, two diagnostic categories (migraine and facial pain) were joined and studied together: the lack of differences between the two groups may be due to the small sample size again. Nevertheless, it seems that the same cortical areas within the pain network are involved in the subjective reality of pain, independently from its type $[3,4]$.

In conclusion, our preliminary results seem to suggest that the description of a pain experience during a pain-free period involves cortical areas similar to those activated during the actual pain perception. Further studies are needed to confirm this hypothesis and to clarify the intriguing subjective dimension of pain. 


\section{References}

1. Hsieh JC, Belfrage M, Stond-Elander $\mathrm{S}$ et al (1995) Central representation of chronic ongoing neuropathic pain studied by positron emission tomography. Pain 63:225-236

2. Porro CA, Cettolo V, Francescato MP, Baraldi P (1998) Temporal and intensity coding of pain in human cortex. $\mathrm{J}$ Neurophysiol 80:3312-3320

3. Derbyshire SWG, Whalley MG, Stenger VA, Oakley DA (2004) Cerebral activation during hypnotically induced and imagined pain. Neuroimage 23:392-401

4. Raij TT, Numminen J, Närvänen $S$, Hiltunen J, Hari R (2005) Brain correlates of subjective reality of physically and hypnotically induced pain. Proc Natl Acad Sci USA 102:2147-2151
5. Melzack R (1975) The McGill Pain Questionnaire: major properties and scoring methods. Pain 1:277-299

6. International Headache Society (2004) The International Classification of Headache Disorders. Cephalalgia 24[Suppl 1]:1-160

7. Oldfield R (1971) The assessment and analysis of handedness: The Edinburgh Inventory. Neuropsychologia 9:97-113

8. Friston KJ, Holmes AP, Worsley KJ et al (1995) Statistical parametric maps in functional imaging: a general linear approach. Hum Brain Mapp 2:189-210
9. Price DD (1999) The phenomenon of pain. In: Price DD (ed) Psychological mechanisms of pain and analgesia. IASP Press, Seattle, WA, pp 1-14

10. Porro CA, Cettolo V, Francescato MP, Baraldi P (2003) Functional activity mapping of the mesial hemispheric wall during anticipation of pain. Neuroimage 19:1738-1747

11. Mongini F, Keller R, Deregibus A et al (2005) Frontal lobe dysfunction in patients with chronic migraine. Psychiatry Res 133:101-106 